Urologe 2016·55:1573-1585

DOI 10.1007/s00120-016-0258-3

Online publiziert: 7. November 2016

(c) Der/die Autor(en) 2016. Dieser Artikel ist eine Open-Access-Publikation.

CrossMark

\author{
T. Reinhold' $\cdot$ C. Dornquast ${ }^{1}$ C. Börgermann ${ }^{2} \cdot$ L. Weißbach ${ }^{3}$ \\ ${ }^{1}$ Institut für Sozialmedizin, Epidemiologie und Gesundheitsökonomie, Charite - Universitätsmedizin, \\ Berlin, Deutschland \\ ${ }^{2}$ Klinik für Urologie, onkologische Urologie und Kinderurologie, Krankenhaus Düren gem. GmbH, Düren, \\ Deutschland \\ ${ }^{3}$ Stiftung Männergesundheit, Berlin, Deutschland
}

\title{
Behandlungskosten des lokal begrenzten Prostatakarzinoms in Deutschland
}

\section{Ökonomische Ergebnisse der HAROW- Beobachtungsstudie}

\begin{abstract}
Das Prostatakarzinom (PCa) ist nach wie vor die häufigste Krebsart bei Männern und die absolute Zahl von Betroffenen wird in den kommenden Jahren weiter steigen. Für die Therapie stehen verschiedene Optionen offen. Obwohl es Hinweise darauf gibt, dass die verfügbaren Behandlungsoptionen vergleichbare Überlebensraten ermöglichen, zeigt die internationale Literatur große Unterschiede in den Folgekosten einzelner Behandlungsarten. Der vorliegende Beitrag thematisiert die ökonomischen Konsequenzen einzelner Therapiestrategien im Versorgungsalltag des deutschen Gesundheitssystems.
\end{abstract}

Das PCa ist mit einer Inzidenz von fast 63.700 (2012) der häufigste Krebs bei Männern in Deutschland [9]. Mögliche Risikofaktoren, die die Entwicklung eines $\mathrm{PCa}$ begünstigen und die Progression beeinflussen, sind Alter, Familiengeschichte und ethnische Herkunft. Mit zunehmendem Alter steigt das PCa-Risiko [10] und es ist zu befürchten, dass die Häufigkeit neuer Erkrankungsfälle in der nächsten Dekade steigen und damit auch die soziale und ökonomische Belastung zunehmen wird.

Die ökonomische Belastung durch das PCa wurde in verschiedenen internationalen Studien untersucht [26].
Aus Japan werden steigende Kosten in den letzten 14 Jahren berichtet [18]. Die Lebenszeitkosten werden für neu diagnostizierte Fälle in den USA mit 4 Mrd. US\$ angegeben. Daraus ergeben sich für das PCa zusätzliche Krankheitskosten pro Patient/Jahr in Höhe von etwa 2600 US\$ [27]. Die gesamten Behandlungskosten werden mit 11,85 Mrd. US\$ im Jahr 2010 angegeben und für 2020 auf etwa 18,53 Mrd. US\$ prognostiziert [17].

Für den lokal begrenzten Tumor gibt es neben palliativen Maßnahmen wie Watchful Waiting (WW) und Hormontherapie (HT) kurative Interventionen wie die aktive Überwachung („Active Surveillance", AS), die perkutane Strahlentherapie (Radiotherapie, RT), die Brachytherapie (BRT) und die radikale Prostatektomie (RP) [5]. Der Unterschied zwischen AS und WW besteht hauptsächlich in der Art des therapeutischen Ansatzes. AS ist eine kurative Behandlungsstrategie, bei der engmaschig überwacht (digital-rektale Untersuchung, PSA-Kontrolle, Rebiopsien) und bei Anzeichen einer Progression oder auf Patientenwunsch hin, invasiv therapiert wird. Dagegen ist WW eine palliative Strategie mit bedarfsorientierter Symptombehandlung, meist durch eine Hormontherapie. Die Wahrscheinlichkeit des gesamt- und des tumorspezifischen Überlebens ist für alle Thera- piearten vergleichbar, die mit einzelnen Behandlungsstrategien assoziierten Gesamtkosten variieren jedoch signifikant $[6,8,13,15,28]$. Nach einer Studie aus den USA reichen die Kosten pro Patient über einen 5-jährigen Nachbeobachtungszeitraum von 7298 US $\$$ unter AS bis hin zu 23.565 US\$ bei RT [15]. In einer schwedischen Studie wurden bei 12-jähriger Nachsorge $34 \%$ höhere Gesamtkosten nach RP im Vergleich $\mathrm{zu}$ WW berichtet [12]. AS wird von anderer Seite als die Option mit den niedrigsten Gesamtkosten über ein 10Jahres-Follow-up beschrieben [8].

Problematisch ist die limitierte Übertragbarkeit dieser Ergebnisse auf den Kontext des deutschen Gesundheitswesens [25]. Deshalb war es das Ziel der vorliegenden Analyse, die mit konkreten Behandlungen des lokal begrenzten $\mathrm{PCa}$ verbundenen Behandlungskosten für Deutschland zu bestimmen. Untersucht wurden die patientenbezogenen Folgekosten für HT, AS, RT, RP sowie WW.

\section{Methodik}

\section{Datenbasis und Studiendesign}

Die ökonomische Analyse der Krankheitskosten basiert auf Daten der HAROW-Studie, einer prospektiven multizentrischen Beobachtungsstudie 
aus den Jahren 2008 bis 2013 [11]. Die an dieser Studie teilnehmenden Ärzte dokumentierten ihre mit der PCa-Behandlung in Zusammenhang stehenden ambulanten Leistungen sowie die von anderen Ärzten verordneten und durchgeführten Behandlungen. Von den insgesamt 259 Studienärzten waren $86 \%$ niedergelassene Urologen. Die Ärzte meldeten ihre Teilnahme nach einem Aufruf im Berufsverbandsorgan Der Urologe und nach einer Informationsveranstaltung auf dem Deutschen Urologenkongress. Einschlusskriterium für Patienten war ein neu diagnostizierter, auf die Prostata begrenzter Tumor $(\leq \mathrm{cT} 2 \mathrm{c})$ ohne Metastasennachweis (N0,M0). Die Patienten wurden gebeten, in Fragebögen Informationen $\mathrm{zu}$ weiterer stationärer und ambulanter Versorgung, zu verordneten Medikamenten, zu Zuzahlungen, Arbeitsunfähigkeit und vorzeitiger Berentung anzugeben. Die Daten wurden halbjährlich erhoben und die Dokumentation erfolgte in Papierform oder auf elektronischem Wege. Da HAROW als reine Beobachtungsstudie konzipiert wurde, gab es keine Vorgaben zu Art und Durchführung der Behandlung.

\section{Analyseperspektive}

Die vorliegende Kostenanalyse reflektiert primär die aus gesellschaftlicher Sicht entstehenden Kosten und wird ergänzt durch eine sekundäre Analyse aus Perspektive der Krankenversicherungsträger, die sich auf die direkten medizinischen Leistungen beschränkt. Aus gesellschaftlicher Sicht ergeben sich zusätzlich indirekte Kosten durch Minderung der volkswirtschaftlichen Produktivität (z. B. bedingt durch Frühberentungen oder Arbeitsunfähigkeit), ebenso wie die vom Patienten zu leistenden Zuzahlungen und Rehabilitationskosten zulasten der gesetzlichen Rentenversicherung.

\section{Kostenbewertung}

Die in dieser Studie analysierten Kosten sollten im Zusammenhang mit der Behandlung des PCa stehen. Es wurden demnach sog. attributable Krankheitskosten berechnet. Entsprechend den dokumentierten Ergebnissen zum Ressour- cenverbrauch und dem Weg des Patienten durch das Gesundheitsversorgungssystem wurden folgende Kostenarten differenziert betrachtet:

- Direkte Kosten

- der ambulanten medizinischen Leistungen seitens der teilnehmenden niedergelassenen Ärzte,

- der von teilnehmenden niedergelassenen Ärzten verordneten, aber von anderen Ärzten erbrachten ambulanten medizinischen Leistungen,

- der stationären Versorgung,

- der stationären Rehabilitation,

- für verordnete Medikation,

- im Rahmen von Zuzahlungen der Patienten.

- Indirekte Kosten

- durch Arbeitsunfähigkeit,

- durch vorzeitige Berentung,

- durch vorzeitigen Tod.

Diese Einzelkostenarten wurden monetär bewertet auf Grundlage der dokumentierten Häufigkeit der Ressourceninanspruchnahme, multipliziert mit standardisierten Bewertungssätzen. Dieses hier gewählte Verfahren wird auch in einschlägigen Publikationen empfohlen, die bei gesundheitsökonomische Analysen auf die Standardisierung der monetären Bewertung der Inanspruchnahme der Gesundheitsversorgung fokussieren $[3,14]$.

\section{Kosten ambulanter medizinischer Leistungen}

Die Bestimmung der Kosten ambulanter medizinischer Leistungen basierte auf den abgerechneten einheitlichen Bewertungsmaßstab(EBM)-Ziffern für Versicherte der gesetzlichen Krankenversicherung (GKV) und Gebührenordnung für Ärzte(GOÄ)-Ziffern für Versicherte privater Krankenversicherungen.

\section{Hospitalisierungskosten}

Um die Hospitalisierungskosten zu ermitteln, wurde zunächst zwischen stationären Aufenthalten zur RP und anderen Hospitalisierungen unterschieden: Für die RP wurden zunächst folgende, für die Abrechnung relevante „diagnosis related groups" (DRGs) bestimmt: M01A, M01B, M02A, M02B, M09A, M09B und
M38Z. Basierend auf den vom InEK (Institut für das Entgeltsystem im Krankenhaus) dokumentierten relativen Häufigkeiten dieser DRGs bei Patienten mit der ICD-Diagnose C61 (bösartige Neubildung der Prostata) wurde das mittlere Relativgewicht von 1,971 durch gewichtete Berechnung ermittelt. Im letzten Schritt wurden die Kosten durch Multiplikation dieses Relativgewichts mit dem mittleren bundesweiten DRG-Basisfallpreis aus dem Jahr 2010 in Höhe von $2935,78 €$ bestimmt. Daraus ergaben sich für einen Krankenhausaufenthalt zur RP durchschnittliche Kosten in Höhe von $5785,61 €$.

Die Kosten weiterer Krankenhausaufenthalte wurden als Produkt der vom Patienten angegebenen Anzahl von Verweildauertagen und einem durchschnittlichen Tagessatz in Höhe von $483,32 €$ errechnet. Dieser Tagessatz wurde bestimmt als Quotient der Gesamtkosten deutscher Krankenhäuser im Jahr 2010 dividiert durch die Gesamtzahl von Krankenhaustagen im selben Jahr. Diese Art der näherungsweisen Kostenbestimmung wurde gewählt, da für von Patienten berichtete Krankenhausaufenthalte keine Abrechnungsinformationen bekannt waren.

\section{Rehabilitationskosten}

Die stationären Rehabilitationskosten wurden aus der dokumentierten Anzahl der Tage, multipliziert mit dem durchschnittlichen Tagessatz von 106,44 €, ermittelt. Dieser Tagessatz ergibt sich aus den Gesamtkosten stationärer rehabilitativer Leistungen dividiert durch die Gesamtzahl von Behandlungstagen 2010. Da Rehabilitationsmaßnahmen für Beschäftigte in Deutschland von der gesetzlichen Rentenversicherung getragen werden, die Rehabilitationskosten für berentete Personen hingegen in der Regel von den gesetzlichen Krankenversicherungssystemen, wurde für die Ermittlung der Rehabilitationskosten nach dem Beschäftigungsstatus der eingeschlossenen Patienten unterschieden.

\section{Medikamentenkosten}

Die verordnete Medikation wurde zunächst entsprechend dem anatomischtherapeutisch-chemischen Klassifikati- 
Hier steht eine Anzeige.

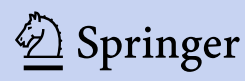


Urologe 2016·55:1573-1585 DOI 10.1007/s00120-016-0258-3

(c) Der/die Autor(en) 2016. Dieser Artikel ist eine Open-Access-Publikation.

\section{T. Reinhold · C. Dornquast · C. Börgermann · L. Weißbach}

\section{Behandlungskosten des lokal begrenzten Prostatakarzinoms in Deutschland. Ökonomische Ergebnisse der HAROW-Beobachtungsstudie}

\section{Zusammenfassung}

Hintergrund. Das Prostatakarzinom (PCa) ist die häufigste Krebsart bei Männern, zu deren Behandlung verschiedene Verfahren zur Verfügung stehen. Unklar sind bislang jedoch die ökonomischen Folgekosten einzelner Behandlungsoptionen im deutschen Versorgungsalltag.

Methodik. Basis der vorliegenden Untersuchung war die zwischen 2008 und 2013 deutschlandweit durchgeführte HAROWBeobachtungsstudie. Die von teilnehmenden Ärzten und Patienten dokumentierten Daten zur Inanspruchnahme von Leistungen des Gesundheitssystems wurden in einer nachgelagerten Kostenanalyse monetär bewertet und anschließend nach Behandlungsarten (Hormontherapie [HT], Active
Surveillance [AS], Radiotherapie [RT], radikale Prostatektomie [RP], Watchful Waiting [WW]) stratifiziert ausgewertet.

Ergebnisse. Insgesamt wurden die Daten von 2672 Patienten ausgewertet. Die Patienten der einzelnen Behandlungsarten waren in Ihren Eigenschaften sehr heterogen. Die höchsten gesellschaftlichen Folgekosten pro Jahr wurden für Patienten nach RP ermittelt (9254 € [95\%-KI 8353-10.154]), wobei der stationäre Aufenthalt zur Operation der Hauptkostentreiber war. Während HT, AS, RT und WW im Hinblick auf die direkten Kosten sehr vergleichbar waren $(805 €[95 \%$ KI 154-1455] für WW bis $1115 €$ [95\%-KI 826-1405] für RT), zeigten sich bei Patienten unter RT die höchsten indirekten Kosten mit
$3928 €(95 \%-K I$ 0-10.675), insbesondere durch Arbeitsunfähigkeit der Patienten. Schlussfolgerung. Die Behandlung des PCa kann erhebliche ökonomische Folgekosten nach sich ziehen, die je nach Behandlungsart stark variieren. Deutlich wird die Notwendigkeit zur Durchführung von gesundheitsökonomischen Langzeitstudien, die behandlungsspezifischen Unterschieden im zeitlichen Auftreten von Kosten besser gerecht werden.

Schlüsselwörter

Kostenanalyse · Prostatakarzinom · Kosten, direkte $\cdot$ Kosten, indirekte $\cdot$ Versorgungsforschung

\section{Treatment costs of localized prostate cancer in Germany. Economic results from the HAROW observational study}

\section{Abstract}

Background. Prostate cancer (PCa) is the most common cancer in men. For medical treatment of $\mathrm{PCa}$, a number of therapies are available. The economic consequences associated with these individual treatment options in routine care in Germany are unclear so far.

Methods. The present analysis was based on the Germany-wide HAROW observational study, which was conducted from 2008-2013. During this study, all participating physicians and involved patients reported and documented individual health care resource consumption. These data were evaluated in monetary terms stratified by treatment regime (hormone therapy, $\mathrm{HT}$; active surveillance, AS; radiotherapy, $\mathrm{RT}$; radical prostatectomy, $\mathrm{RP}$; watchful waiting, WW).

Results. Overall, the data of 2672 patients were available for analysis. Based on the observational study design, the included patient groups were heterogeneous in their baseline characteristics. The annual total costs from the societal perspective were the largest for patient undergoing RP (9254 €; $95 \% \mathrm{Cl} 8353-10,154)$, mainly driven by the costs for the initial hospital stay for surgery. $\mathrm{HT}, \mathrm{AS}, \mathrm{RT}$, and WW seem to be comparable in terms of direct costs, ranging from $805 €$ (95\% Cl 154-1455) for WW up to $1115 €$ (95\% Cl 826-1405) for RT. The highest indirect costs were observed for patients receiving
RT (3928 €; $95 \%$ Cl 0-10,675), which can be justified by the frequent incapacity to work in this patient group.

Conclusion. The treatment of prostate cancer can lead to significant economic follow-up costs which vary greatly depending on the type of treatment. The analysis indicates a need for the implementation of a long-term health economic study in the future, which will be more suitable to show treatmentspecific differences in the temporal occurrence of costs.

Keywords

Cost-analysis - Prostate cancer - Costs, direct . Indirect costs $\cdot$ Health services research
onssystem(ATC)-Code als PCa-bezogen klassifiziert. Danach wurden die Kosten der relevanten Medikation durch Multiplikation der Apothekenpreise [4] mit der Häufigkeit der dokumentierten Verschreibungen ermittelt.

\section{Patientenzuzahlungen}

Patientenzuzahlungen wurden von den Patienten direkt abgefragt und differenziert nach Zuzahlungen für Medikamen- te, Krankenhausaufenthalte und stationäre Rehabilitation betrachtet.

\section{Indirekte Kosten}

Die Bestimmung der indirekten Kosten basiert auf dem sog. Humankapitalansatz. Dieser gehört zu den weitreichendsten methodischen Herangehensweisen um gesellschaftliche Produktivitätsverluste monetär zu bestimmen [16]. Als mittlere Kosten pro Arbeitsunfähigkeitstag wurden $82,94 €$ zugrunde gelegt. Dies entspricht dem täglichen mittleren deutschen Bruttoinlandsprodukt pro Person im Jahr 2010. Zur Bestimmung der indirekten Kosten durch vorzeitigen Tod oder Frühberentung wurde zunächst die Differenz zwischen aktuellem Alter bei vorzeitigem Tod oder Frühberentung und durchschnittlichem Renteneintrittsalter von Männern im Jahr 2010 (63,75 Jahre) errechnet. Anschließend wurde jedes verlorene Erwerbstätigkeitsjahr mit $30.295 €$ bewertet (Brut- 


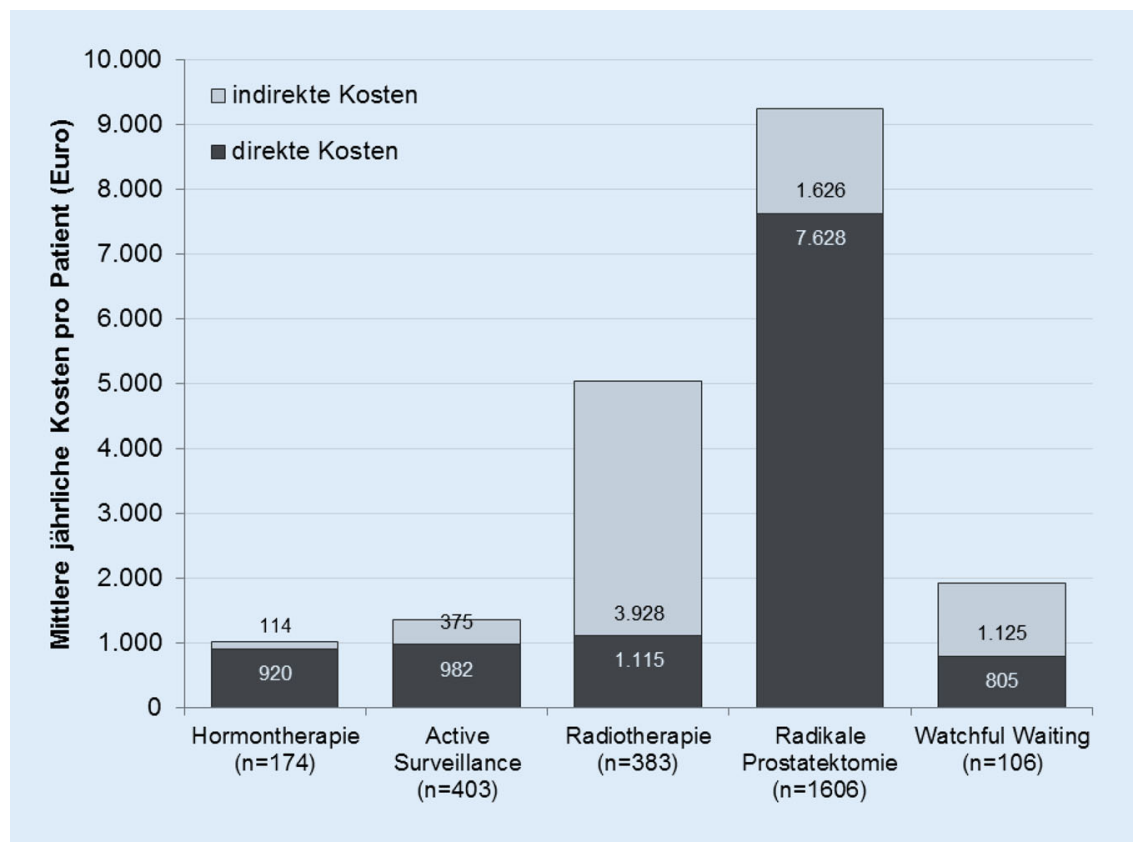

Abb. 1 ॥ Mittlere jährliche Kosten pro Patient aus gesellschaftlicher Perspektive

toinlandsprodukt pro Person im Jahr 2010).

\section{Statistik}

Da es sich bei der zugrunde liegenden HAROW-Studie um eine reine Beobachtungsstudie handelt, war auch die vorliegende gesundheitsökonomische Analyse vornehmlich deskriptiv ausgerichtet. Alle patientenbezogenen jährlichen attributablen Krankheitskosten wurden als Mittelwerte mit $95 \%$-Konfidenzintervall dargestellt.

Um die patientenbezogenen jährlichen Kosten detailreicher darzustellen, umfasste die Berechnung der durchschnittlichen Kosten zunächst alle einer Behandlungsgruppe zugeordneten Patienten, einschließlich derer, die de facto keine Kosten verursachten. Um darüber hinaus Informationen über die mittleren Kosten der Patienten zu erhalten, für die auch eine konkrete Ressourceninanspruchnahme dokumentiert war, erfolgte eine weitere Mittelwertberechnung nur unter Einbeziehung genau jener betroffener Patienten.

Alle Analysen wurden unter Verwendung der PASW-Statistik Version 22.0.0.1 (SPSS Inc., Chicago, Ill.) durchgeführt. und 5,8 ng/ml bei Patienten in der ASGruppe.

\section{Ergebnisse zu Behandlungskosten}

\section{Radikale Prostatektomie}

Die RP ist hinsichtlich der Gesamtkosten mit der höchsten Last für die Gesellschaft verbunden (• Abb. 1). Für diese Gruppe belaufen sich die jährlichen Gesamtkosten pro Patient auf $9254 €$ (95\%KI 8353-10.154) (• Tab. 2). Mit $4518 €$ (95\% KI-4149-4887) kann der größte Teil der Kosten der ersten Hospitalisierung zur Operation zugeordnet werden. Die Gesamtkosten des Krankenhausaufenthaltes in Höhe von $6599 €(95 \%$ KI 6039-7185) lassen sich mit weiteren stationären Behandlungen von insgesamt 800 Patienten in dieser Gruppe erklären (• Tab. 3). Zusätzlich ermittelten wir indirekte jährliche Kosten in Höhe von $1626 €$ (95\%-KI 1210-2042). Im Vergleich mit den anderen Behandlungsgruppen scheinen diese ein Effekt des vergleichsweise jungen Alters und der häufigeren Erwerbstätigkeit zu sein. 296 Patienten verursachten indirekte Kosten wegen Frühberentung, vorzeitigen Todes oder Arbeitsunfähigkeit. Wenn nur diese Patienten betrachtet werden, erhöhen sich die mittleren indirekten Kosten pro Patient und Jahr auf $8821 €$ (95\%-KI 6751-10.890). Die Zuzahlungen, hauptsächlich zum stationären Aufenthalt oder zur stationären Rehabilitation, beliefen sich im Mittel auf $127 €$ (95\%-KI 101-153). Aus Sicht der Krankenversicherung lagen die direkten Gesamtkosten der Patienten unter RP pro Jahr bei $7125 €$ (95\%-KI 6535-7715).

\section{Radiotherapie}

Die gesamten direkten und indirekten Kosten für RT-Patienten lagen bei $5043 €$ (95\%-KI 0-11.792; • Tab. 2). Der Großteil der Gesamtkosten entsteht durch den gesellschaftlichen Produktivitätsverlust. Diese indirekten Kosten machen $79 \%$ der Gesamtkosten oder $3928 €$ aus (95\%-KI 0-10.575). Der Betrag scheint vergleichsweise hoch, erklärt sich aber aus der relativ geringen absoluten Zahl von 28 betroffenen Patienten ( $\bullet$ Tab. 3). Ein Patient in dieser Gruppe verstarb vorzeitig und verursachte somit indirekte 
Tab. 1 Baselinecharakteristika der eingeschlossenen Patienten zu Beobachtungsbeginn

\begin{tabular}{|c|c|c|c|c|c|}
\hline & $\begin{array}{l}\text { Hormon- } \\
\text { therapie }\end{array}$ & $\begin{array}{l}\text { Active Sur- } \\
\text { veillance }\end{array}$ & $\begin{array}{l}\text { Radio- } \\
\text { therapie }\end{array}$ & $\begin{array}{l}\text { Radikale } \\
\text { Prostatek- } \\
\text { tomie }\end{array}$ & $\begin{array}{l}\text { Watchful } \\
\text { Waiting }\end{array}$ \\
\hline$n$ & 174 & 403 & 383 & 1.606 & 106 \\
\hline Alter (Jahre, MW [SA]) & $75,3(6,0)$ & $68,0(7,0)$ & $70,1(6,6)$ & $64,8(6,8)$ & $72,7(7,4)$ \\
\hline $\begin{array}{l}\text { Charlson-Komorbiditäts- } \\
\text { index (MW [SA]) }\end{array}$ & $3,80(1,4)$ & $2,72(1,2)$ & $3,05(1,3)$ & $2,33(1,1)$ & $3,32(1,3)$ \\
\hline \multicolumn{6}{|c|}{ Tumorkategorie (\% der Patienten) } \\
\hline $\mathrm{T} 1 \mathrm{a} / 1 \mathrm{~b}$ & 4,6 & 20,8 & 1,6 & 11,0 & 11,0 \\
\hline T1c & 48,3 & 64,0 & 54,2 & 56,2 & 34,0 \\
\hline T2a & 6,3 & 10,9 & 15,2 & 11,3 & 10,4 \\
\hline $\mathrm{T} 2 \mathrm{~b}$ & 11,5 & 2,2 & 11,3 & 10,0 & 5,7 \\
\hline $\mathrm{T} 2 \mathrm{C}$ & 29,3 & 2,0 & 17,8 & 19,9 & 10,4 \\
\hline $\begin{array}{l}\text { PSA-Wert (ng/ml, MW } \\
\text { [SA]) }\end{array}$ & $20,9(42,6)$ & $5,8(3,5)$ & $10,8(20,8)$ & $8,8(7,8)$ & $6,8(4,6)$ \\
\hline \multicolumn{6}{|c|}{ PSA-Dichte (\% der Patienten) } \\
\hline$<0,2$ & 29,3 & 72,5 & 39,7 & 46,3 & 43,4 \\
\hline$\geq 0,2$ & 55,2 & 20,3 & 37,9 & 42,8 & 31,1 \\
\hline n.a. & 15,5 & 7,2 & 22,5 & 10,8 & 25,5 \\
\hline \multicolumn{6}{|c|}{ Gleason-Score (\% der Patienten) } \\
\hline $4-6$ & 42,5 & 92,8 & 57,7 & 51,7 & 47,2 \\
\hline $7 a$ & 21,8 & 5,5 & 22,2 & 28,3 & 18,9 \\
\hline $7 b$ & 12,1 & 0,2 & 11,0 & 9,3 & 3,8 \\
\hline $8-10$ & 23,0 & 0,2 & 8,1 & 9,3 & 1,9 \\
\hline n.a. & 0,6 & 1,2 & 1,0 & 1,3 & 28,3 \\
\hline
\end{tabular}

Kosten von $95.911 €$. Weitere 3 Patienten wurden PCa-bedingt frühberentet; die mittleren indirekten Pro-Kopf-Kosten für diese Patienten lagen bei $440.201 €$. Betrachtet man die vergleichsweise hohen indirekten Kosten in der RT-Gruppe, zeigt sich, dass sie stark von diesen sog. Ausreißern beeinflusst sind. Die direkten Gesamtkosten pro Patient und Jahr lagen bei $1115 €$ (95\%-KI 826-1405). Davon entfallen $983 €(95 \%-K I ~ 717-1249) \mathrm{zu}$ Lasten der Krankenversicherung.

\section{Hormontherapie}

HT-Patienten verursachten mittlere Gesamtkosten in Höhe von $1033 €$ (95 \%-KI 698-1369; • Tab. 2). Im Gegensatz zu den RT-Patienten lag der Anteil der indirekten Kosten durch Produktivitätsverluste bei nur $11 \%$ bzw. $114 €$ (95\%-KI 0-302). Die Patienten der HT-Gruppe waren am ältesten und größtenteils nicht mehr erwerbstätig. Die direkten Kosten in dieser Gruppe wurden in erster Linie durch die Ausgaben für Medikamente in Höhe von $458 €$ (95\%-KI 261-655) pro Per- direkten Kosten von $911 €(95 \%-\mathrm{KI}$ 723-1100) pro Person.

\section{Watchful Waiting}

WW ist eine weitere weniger invasive Behandlungsoption die, einschließlich der indirekten Kosten, Gesamtkosten in Höhe von $1929 €$ (95\%-KI 1279-4069) verursacht (-Tab. 2). Wie bei den anderen therapeutischen Optionen (außer der RP) waren die indirekten Kosten nur einer vergleichsweise geringen Anzahl betroffener Patienten zuzuordnen (• Tab. 3). Die direkten Kosten von $805 €$ (95\%-KI 154-1455) waren vor allem durch Krankenhausaufenthalte bedingt. Obwohl keiner der WW-Patienten im Verlauf der Studie eine RP hatte, benötigten 18 Patienten eine stationäre Behandlung. Abhängig von deren Dauer beliefen sich die mittleren Kosten dieser 18 Patienten auf $3254 €$. Bezogen auf alle Patienten der WW-Gruppe resultierten durchschnittlich jährliche Hospitalisierungskosten von $553 €$ (95\%-KI 0-1148) pro Patient. Aus Sicht der Krankenversicherung lagen die jährlichen mittleren Kosten bei $745 €$ (95\%-KI 101-1389) pro Patient.

\section{Diskussion} tienten wurde Medikamenteneinnahme dokumentiert. Für diese Patienten lagen die Arzneimittelkosten ohne Berücksichtigung der Zuzahlungen bei $759 €$ (95\%KI 0-1073; - Tab. 3). Die jährlichen Gesamtkosten zulasten der Krankenversicherung beliefen sich auf $878 €(95 \%$-KI 602-1153) pro Person.

\section{Active Surveillance}

Die Gesamtkosten dieser therapeutischen Option lagen bei jährlich $1358 €$ (95\%-KI 1004-1711) pro Person (• Tab. 2). Davon entfielen $982 €$ (95\%KI 787-1178) pro Person auf direkte Kosten, vor allem aufgrund stationärer Versorgung. 64 Patienten (16\%) hatten einen stationären Aufenthalt zur RP und weitere 80 Patienten benötigten im Verlauf der Beobachtung zusätzliche stationäre Behandlungen (• Tab. 3). Der Anteil indirekter Kosten lag bei $28 \%$ der Gesamtkosten. Aus Sicht der Krankenversicherung führte AS jährlich zu
Unsere Daten weisen auf stark differierende Folgekosten der verschiedenen Behandlungsoptionen des lokal begrenzten PCa hin. Aus ökonomischer Sicht bestätigen sich damit im Wesentlichen die Erkenntnisse aus anderen Ländern $[8,12$, $15]$.

\section{Methodische Aspekte}

Einige der Analyse zugrundeliegendem methodischen Aspekte führen zu Einschränkungen und sollten bei der Interpretation der Ergebnisse Berücksichtigung finden.

So ergibt sich eine Limitation der Aussagekraft der gefundenen Ergebnisse durch die mittlere Nachbeobachtungszeit von 2,5 Jahren. Aufgrund dieser vergleichsweise kurzen Beobachtungsdauer können möglicherweise auftretende Mehrkosten für Folgetherapien bzw. Komplikationsmanagement $\mathrm{zu}$ einem späteren Zeitpunkt nicht eruiert werden. 
Hier steht eine Anzeige.

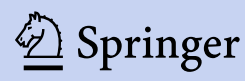


Tab. 2 Mittlere jährliche Kosten pro Patient (bezogen auf alle Patienten der jeweiligen Gruppe)

\section{\begin{tabular}{l|l|l|l} 
Hormontherapie Active Surveillance & Radiotherapie & Radikale Prostatek- Watchful Waiting
\end{tabular}}

\begin{tabular}{|c|c|c|c|c|c|c|c|c|c|c|}
\hline \multirow[b]{2}{*}{ Kostenart } & \multicolumn{2}{|c|}{ Hormontherapie } & \multicolumn{2}{|c|}{ Active Surveillance } & \multicolumn{2}{|c|}{ Radiotherapie } & \multicolumn{2}{|c|}{$\begin{array}{l}\text { Radikale Prostatek- } \\
\text { tomie }\end{array}$} & \multicolumn{2}{|c|}{ Watchful Waiting } \\
\hline & $n$ & MW (95 \%-KI) & $n$ & MW (95 \%-KI) & $n$ & MW (95 \%-KI) & $n$ & MW (95 \%-KI) & $n$ & MW (95 \%-KI) \\
\hline Frühberentung & 174 & $0(0-0)$ & 403 & $107,91(0-254)$ & 383 & $\begin{array}{l}3448,05 \\
(0-10178)\end{array}$ & 1606 & $\begin{array}{l}334,57 \\
(179-490)\end{array}$ & 106 & $301,33(0-747)$ \\
\hline $\begin{array}{l}\text { Vorzeitigte Sterblich- } \\
\text { keit }\end{array}$ & 174 & $95,2(0-282)$ & 403 & $114,25(0-338)$ & 383 & $250,42(0-741)$ & 1606 & $201,09(0-441)$ & 106 & - \\
\hline Arbeitsunfähigkeit & 174 & $18,51(0-45)$ & 403 & $153,2(27-280)$ & 383 & $229,52(56-403)$ & 1606 & $\begin{array}{l}1090,05 \\
(794-1387)\end{array}$ & 106 & $823,63(0-2294)$ \\
\hline $\begin{array}{l}\text { Summe: indirekte } \\
\text { Kosten }\end{array}$ & 174 & $113,7(0-302)$ & 403 & $\begin{array}{l}375,36 \\
(81-670)\end{array}$ & 383 & $\begin{array}{l}3927,99 \\
(0-10675)\end{array}$ & 1606 & $\begin{array}{l}1625,71 \\
(1210-2042)\end{array}$ & 106 & $\begin{array}{l}1124,96 \\
(0-2656)\end{array}$ \\
\hline Arzneimittelkosten & 174 & $\begin{array}{l}458,15 \\
(261-655)\end{array}$ & 403 & $33,86(12-56)$ & 383 & $\begin{array}{l}146,98 \\
(103-191)\end{array}$ & 1606 & $46,76(37-56)$ & 106 & $65,41(11-120)$ \\
\hline $\begin{array}{l}\text { Hospitalisierung wg. } \\
\text { Prostatektomie }\end{array}$ & 174 & - & 403 & $\begin{array}{l}358,85 \\
(258-460)\end{array}$ & 383 & - & 1606 & $\begin{array}{l}4517,96 \\
(4149-4887)\end{array}$ & 106 & - \\
\hline $\begin{array}{l}\text { Weitere Hospitalisie- } \\
\text { rungen }\end{array}$ & 174 & $\begin{array}{l}296,01 \\
(128-464)\end{array}$ & 403 & $\begin{array}{l}343,52 \\
(214-473)\end{array}$ & 383 & $\begin{array}{l}568,14 \\
(343-794)\end{array}$ & 1606 & $\begin{array}{l}2080,59 \\
(1754-2408)\end{array}$ & 106 & $552,59(0-1148)$ \\
\hline $\begin{array}{l}\text { Summe: Hospitalisie- } \\
\text { rungen }\end{array}$ & 174 & $\begin{array}{l}296,01 \\
(128-464)\end{array}$ & 403 & $\begin{array}{l}702,37 \\
(531-874)\end{array}$ & 383 & $\begin{array}{l}568,14 \\
(343-794)\end{array}$ & 1606 & $\begin{array}{l}6598,55 \\
(6039-7158)\end{array}$ & 106 & $552,59(0-1148)$ \\
\hline $\begin{array}{l}\text { Ambulante Studien- } \\
\text { arztkosten }\end{array}$ & 174 & $71,51(60-83)$ & 403 & $94,09(86-102)$ & 383 & $85,85(65-107)$ & 1606 & $57,28(53-62)$ & 106 & $82,5(66-99)$ \\
\hline $\begin{array}{l}\text { Ambulante weitere } \\
\text { Arztkosten }\end{array}$ & 174 & $2,32(1-4)$ & 403 & $4,22(2-6)$ & 383 & $1,51(1-2)$ & 1606 & $7,36(0-15)$ & 106 & $1,27(1-2)$ \\
\hline $\begin{array}{l}\text { Summe: Ambulante } \\
\text { Arztkosten }\end{array}$ & 174 & $73,84(63-85)$ & 403 & $98,32(90-107)$ & 383 & $87,36(66-108)$ & 1606 & $64,65(55-74)$ & 106 & $83,77(67-100)$ \\
\hline $\begin{array}{l}\text { Rehabilitation (Kran- } \\
\text { kenversicherung) }\end{array}$ & 174 & $49,74(23-77)$ & 403 & $76,65(37-116)$ & 383 & $180,18(93-268)$ & 1606 & $\begin{array}{l}415,22 \\
(341-489)\end{array}$ & 106 & $42,85(5-81)$ \\
\hline $\begin{array}{l}\text { Rehabilitation (Renten- } \\
\text { versicherung) }\end{array}$ & 174 & $3,51(0-10)$ & 403 & $34,11(16-52)$ & 383 & $82,16(0-168)$ & 1606 & $\begin{array}{l}375,65 \\
(278-473)\end{array}$ & 106 & $37,28(0-78)$ \\
\hline $\begin{array}{l}\text { Summe: Rehabilitati- } \\
\text { onskosten }\end{array}$ & 174 & $53,25(25-81)$ & 403 & $110,76(68-154)$ & 383 & $\begin{array}{l}262,35 \\
(141-384)\end{array}$ & 1606 & $\begin{array}{l}790,87 \\
(671-910)\end{array}$ & 106 & $80,13(25-135)$ \\
\hline $\begin{array}{l}\text { Patientenzuzahlung: } \\
\text { Medikamente }\end{array}$ & 174 & $34,62(15-54)$ & 403 & $19,49(5-34)$ & 383 & $24,25(15-34)$ & 1606 & $43,92(33-54)$ & 106 & $16,45(0-35)$ \\
\hline $\begin{array}{l}\text { Patientenzuzahlung: } \\
\text { Hospitalisierung }\end{array}$ & 174 & $2,83(0-5)$ & 403 & $13,5(5-22)$ & 383 & $13,23(1-25)$ & 1606 & $63,93(44-84)$ & 106 & $5,12(0-12)$ \\
\hline $\begin{array}{l}\text { Patientenzuzahlung: } \\
\text { Rehabilitation }\end{array}$ & 174 & $0,96(0-2)$ & 403 & $3,98(1-7)$ & 383 & $13,17(3-23)$ & 1606 & $19,17(14-25)$ & 106 & $1,04(0-3)$ \\
\hline $\begin{array}{l}\text { Summe: Patientenzu- } \\
\text { zahlung }\end{array}$ & 174 & $38,42(18-58)$ & 403 & $36,97(20-54)$ & 383 & $50,65(32-70)$ & 1606 & $\begin{array}{l}127,02 \\
(101-153)\end{array}$ & 106 & $22,61(3-42)$ \\
\hline $\begin{array}{l}\text { Summe: direkte } \\
\text { Kosten }\end{array}$ & 174 & $\begin{array}{l}919,66 \\
(636-1203)\end{array}$ & 403 & $\begin{array}{l}982,28 \\
(787-1178)\end{array}$ & 383 & $\begin{array}{l}1115,48 \\
(826-1405)\end{array}$ & 1606 & $\begin{array}{l}7627,84 \\
(6967-8288)\end{array}$ & 106 & $\begin{array}{l}804,51 \\
(154-1455)\end{array}$ \\
\hline $\begin{array}{l}\text { Gesamtkosten } \\
\text { gesellschaftliche } \\
\text { Perspektive }^{\mathrm{a}}\end{array}$ & 174 & $\begin{array}{l}1033,36 \\
(698-1369)\end{array}$ & 403 & $\begin{array}{l}1357,64 \\
(1004-1711)\end{array}$ & 383 & $\begin{array}{l}5043,47 \\
(0-11792)\end{array}$ & 1606 & $\begin{array}{l}9253,55 \\
(8353-10154)\end{array}$ & 106 & $\begin{array}{l}1929,48 \\
(0-4069)\end{array}$ \\
\hline $\begin{array}{l}\text { Gesamtkosten Kran- } \\
\text { kenkassenperspektive }^{\mathrm{t}}\end{array}$ & 174 & $\begin{array}{l}877,73 \\
(602-1153)\end{array}$ & 403 & $\begin{array}{l}911,19 \\
(723-1100)\end{array}$ & 383 & $\begin{array}{l}982,67 \\
(717-1249)\end{array}$ & 1606 & $\begin{array}{l}7125,18 \\
(6535-7715)\end{array}$ & 106 & $\begin{array}{l}744,63 \\
(101-1389)\end{array}$ \\
\hline \multicolumn{11}{|c|}{$\begin{array}{l}\text { MW Mittelwert, } 95 \% \text {-KI } 95 \% \text {-Konfidenzintervall } \\
{ }^{a} \text { Gesellschaftliche Perspektive = Summe indirekte Kosten + Summe direkte Kosten } \\
{ }^{b} \text { Krankenkassenperspektive = Summe direkte Kosten - Summe Patientenzuzahlung - Rehabilitationskosten (Rentenversicherung) } \\
\text { Kursiv Unterkategorien einer übergeordneten Kostenart }\end{array}$} \\
\hline
\end{tabular}


Tab. 3 Mittlere jährliche Kosten pro Patient (bezogen auf betroffene Patienten der jeweiligen Gruppe)

\begin{tabular}{|c|c|c|c|c|c|c|c|c|c|c|}
\hline \multirow[b]{2}{*}{ Kostenart } & \multicolumn{2}{|c|}{ Hormontherapie } & \multicolumn{2}{|c|}{ Active Surveillance } & \multicolumn{2}{|c|}{ Radiotherapie } & \multicolumn{2}{|c|}{$\begin{array}{l}\text { Radikale Prostatek- } \\
\text { tomie }\end{array}$} & \multicolumn{2}{|c|}{ Watchful Waiting } \\
\hline & $n$ & MW (95 \%-KI) & $n$ & MW (95 \%-KI) & $n$ & MW (95 \%-KI) & $n$ & MW (95 \%-KI) & $n$ & MW (95 \%-KI) \\
\hline Frühberentung & 0 & - & 3 & $\begin{array}{l}14.496,1 \\
(1.331-27.661)\end{array}$ & 3 & $\begin{array}{l}440.201,2 \\
(0-1.297 .528)\end{array}$ & 28 & $\begin{array}{l}19.189,8 \\
(13.636-24.744)\end{array}$ & 2 & $\begin{array}{l}15.970,5 \\
(3.971-27.970)\end{array}$ \\
\hline $\begin{array}{l}\text { Vorzeitigte Sterblich- } \\
\text { keit }\end{array}$ & 1 & $\begin{array}{l}16.564,1 \\
(16.564-16.564)\end{array}$ & 1 & $\begin{array}{l}46.043,6 \\
(46.044-46.044)\end{array}$ & 1 & $\begin{array}{l}95.911,4 \\
(95.911-95.911)\end{array}$ & 4 & $\begin{array}{l}80.739,4 \\
(17.157-144.322)\end{array}$ & 0 & $0(0-0)$ \\
\hline Arbeitsunfähigkeit & 2 & $\begin{array}{l}1.610,2 \\
(850-2.370)\end{array}$ & 15 & $\begin{array}{l}4.115,9 \\
(1.305-6.926)\end{array}$ & 25 & $\begin{array}{l}3.516,2 \\
(1.175-5.857)\end{array}$ & 282 & $\begin{array}{l}6.207,9 \\
(4.650-7.766)\end{array}$ & 4 & $\begin{array}{l}21.826,2 \\
(0-59.491)\end{array}$ \\
\hline $\begin{array}{l}\text { Summe: indirekte } \\
\text { Kosten }\end{array}$ & 3 & $\begin{array}{l}6.594,8 \\
(0-16.375)\end{array}$ & 19 & $\begin{array}{l}7.961,6 \\
(2.660-13.264)\end{array}$ & 28 & $\begin{array}{l}53.729,3 \\
(0-145.541)\end{array}$ & 296 & $\begin{array}{l}8.820,6 \\
(6.751-10.890)\end{array}$ & 6 & $\begin{array}{l}19.874,3 \\
(0-44.018)\end{array}$ \\
\hline Arzneimittelkosten & 105 & $759,2(0-1.073)$ & 135 & $101,1(0-165)$ & 240 & $234,6(0-303)$ & 607 & $123,7(0-148)$ & 28 & $247,6(0-440)$ \\
\hline $\begin{array}{l}\text { Hospitalisierung wg. } \\
\text { Prostatektomie }\end{array}$ & 0 & - & 64 & $\begin{array}{l}2.259,6 \\
(1.879-2.641)\end{array}$ & 0 & - & 1466 & $\begin{array}{l}4.949,4 \\
(4.552-5.347)\end{array}$ & 0 & - \\
\hline $\begin{array}{l}\text { Weitere Hospitalisie- } \\
\text { rungen }\end{array}$ & 23 & $\begin{array}{l}2.239,3 \\
(1.279-3.200)\end{array}$ & 80 & $\begin{array}{l}1.730,5 \\
(1.171-2.290)\end{array}$ & 100 & $\begin{array}{l}2.176 \\
(1.391-2.961)\end{array}$ & 800 & $\begin{array}{l}4.176,8 \\
(3.553-4.800)\end{array}$ & 18 & $\begin{array}{l}3.254,2 \\
(0-6.557)\end{array}$ \\
\hline $\begin{array}{l}\text { Summe: Hospitalisie- } \\
\text { rungen }\end{array}$ & 23 & $\begin{array}{l}2.239,3 \\
(1.279-3.200)\end{array}$ & 103 & $\begin{array}{l}2.748,1 \\
(2.255-3.241)\end{array}$ & 100 & $\begin{array}{l}2.176 \\
(1.391-2.961)\end{array}$ & 1541 & $\begin{array}{l}6.876,9 \\
(6.298-7.456)\end{array}$ & 18 & $\begin{array}{l}3.254,2 \\
(0-6.557)\end{array}$ \\
\hline $\begin{array}{l}\text { Ambulante Studien- } \\
\text { arztkosten }\end{array}$ & 138 & $90,2(78-102)$ & 380 & $99,8(92-108)$ & 292 & $112,6(86-140)$ & 1003 & $91,7(85-98)$ & 97 & $90,2(73-107)$ \\
\hline $\begin{array}{l}\text { Ambulante weitere } \\
\text { Arztkosten }\end{array}$ & 33 & $12,3(7-18)$ & 100 & $17(9-25)$ & 68 & $8,5(7-10)$ & 249 & $47,5(-3-98)$ & 15 & $9(7-11)$ \\
\hline $\begin{array}{l}\text { Summe: Ambulante } \\
\text { Arztkosten }\end{array}$ & 144 & $89,2(77-101)$ & 381 & $104(95-113)$ & 299 & $111,9(86-138)$ & 1010 & $102,8(88-117)$ & 99 & $89,7(73-107)$ \\
\hline $\begin{array}{l}\text { Rehabilitation (Kran- } \\
\text { kenversicherung) }\end{array}$ & 14 & $618,2(496-750)$ & 27 & $\begin{array}{l}1.144,1 \\
(868-1.572)\end{array}$ & 40 & $\begin{array}{l}1.725,3 \\
(934-2.400)\end{array}$ & 440 & $\begin{array}{l}1.515,6 \\
(1.278-1.759)\end{array}$ & 5 & $\begin{array}{l}908,4 \\
(632-1.159)\end{array}$ \\
\hline $\begin{array}{l}\text { Rehabilitation (Renten- } \\
\text { versicherung) }\end{array}$ & 1 & $611,1(611-611)$ & 17 & $\begin{array}{l}808,6 \\
(609-1.008)\end{array}$ & 9 & $\begin{array}{l}3.496,6 \\
(437-6.556)\end{array}$ & 317 & $\begin{array}{l}1.903,1 \\
(1.446-2.360)\end{array}$ & 4 & $988(398-1.578)$ \\
\hline $\begin{array}{l}\text { Summe: Rehabilitati- } \\
\text { onskosten }\end{array}$ & 15 & $\begin{array}{l}617,7 \\
(495-740)\end{array}$ & 44 & $\begin{array}{l}1.014,5 \\
(739-1.290)\end{array}$ & 49 & $\begin{array}{l}2.050,6 \\
(1.259-2.842)\end{array}$ & 757 & $\begin{array}{l}1.677,9 \\
(1.440-1.916)\end{array}$ & 9 & $\begin{array}{l}943,8 \\
(668-1.220)\end{array}$ \\
\hline $\begin{array}{l}\text { Patientenzuzahlung: } \\
\text { Medikamente }\end{array}$ & 105 & $57,4(52-89)$ & 135 & $58,2(51-101)$ & 240 & $38,7(37-54)$ & 607 & $116,2(66-143)$ & 28 & $62,3(60-129)$ \\
\hline $\begin{array}{l}\text { Patientenzuzahlung: } \\
\text { Hospitalisierung }\end{array}$ & 23 & $21,4(4-38)$ & 80 & $68(28-108)$ & 100 & $50,7(6-96)$ & 800 & $128,3(89-168)$ & 18 & $30,2(0-67)$ \\
\hline $\begin{array}{l}\text { Patientenzuzahlung: } \\
\text { Rehabilitation }\end{array}$ & 15 & $11,2(1-21)$ & 44 & $36,4(14-59)$ & 49 & $102,9(31-175)$ & 757 & $40,7(29-52)$ & 9 & $12,2(0-31)$ \\
\hline $\begin{array}{l}\text { Summe: Patientenzu- } \\
\text { zahlung }\end{array}$ & 111 & $60,2(30-91)$ & 172 & $86,6(47-126)$ & 269 & $72,1(45-99)$ & 1000 & 204 (164-244) & 40 & $59,9(11-109)$ \\
\hline $\begin{array}{l}\text { Summe: direkte } \\
\text { Kosten }\end{array}$ & 174 & $\begin{array}{l}919,7 \\
(636-1.203)\end{array}$ & 402 & $\begin{array}{l}984,7 \\
(789-1.180)\end{array}$ & 381 & $\begin{array}{l}1.121,3 \\
(830-1.412)\end{array}$ & 1606 & $\begin{array}{l}7.627,8 \\
(6.967-8.288)\end{array}$ & 106 & $\begin{array}{l}804,5 \\
(154-1.455)\end{array}$ \\
\hline $\begin{array}{l}\text { Gesamtkosten } \\
\text { gesellschaftliche } \\
\text { Perspektive }^{a}\end{array}$ & 174 & $\begin{array}{l}1.033,4 \\
(750-1.369)\end{array}$ & 403 & $\begin{array}{l}1.357,6 \\
(1.162-1.711)\end{array}$ & 383 & $\begin{array}{l}5.043,5 \\
(4.753-11.792)\end{array}$ & 1606 & $\begin{array}{l}9.253,6 \\
(8.593-10.154)\end{array}$ & 106 & $\begin{array}{l}1.929,5 \\
(1.279-4.069)\end{array}$ \\
\hline $\begin{array}{l}\text { Gesamtkosten Kran- } \\
\text { kenkassenperspektive }^{\mathrm{b}}\end{array}$ & 174 & $\begin{array}{l}877,7 \\
(602-1.153)\end{array}$ & 401 & $\begin{array}{l}915,7 \\
(726-1.105)\end{array}$ & 379 & $993(724-1.262)$ & 1606 & $\begin{array}{l}7.125,2 \\
(6.535-7.715)\end{array}$ & 105 & $\begin{array}{l}751,7 \\
(102-1.402)\end{array}$ \\
\hline \multicolumn{11}{|c|}{$\begin{array}{l}\text { MW Mittelwert; } 95 \% \text {-KI } 95 \% \text {-Konfidenzintervall } \\
{ }^{a} \text { Gesellschaftliche Perspektive = Summe indirekte Kosten + Summe direkte Kosten } \\
{ }^{b} \text { Krankenkassenperspektive = Summe direkte Kosten - Summe Patientenzuzahlung - Rehabilitationskosten (Rentenversicherung) } \\
\text { Kursiv Unterkategorien einer übergeordneten Kostenart }\end{array}$} \\
\hline
\end{tabular}


Dies gilt besonders für Therapiekonzepte wie AS, bei denen aufwendige Leistungen oft erst $\mathrm{zu}$ einem späteren Zeitpunkt notwendig werden. Hier kann unterstellt werden, dass die vorliegenden Kostenergebnisse als eher konservativ einzuschätzen sind.

Zudem ergeben sich Limitationen im Rahmen der eingeschlossenen Kostenarten. Entsprechend der zugrundeliegenden Methodik wurden nicht alle tatsächlich auftretenden Kosten berücksichtigt. Das gilt beispielsweise für Infrastrukturbzw. Investitionskosten bei der Strahlentherapie oder robotergestützter Operationen. Auf das anteilige Umlegen dieser Kosten auf den einzelnen Patienten wurde in der vorliegenden Studie bewusst verzichtet, da hierzu valide Informationen zur wahrscheinlichen Lebensdauer und zur Auslastung der Gerätschaften hätten erhoben werden müssen. Zudem würde sich die patientenbezogene Kostenzuteilung mit steigender Auslastung im Zeitverlauf stetig ändern, die Daten wären sehr zentrumspezifisch und würden damit eine Verallgemeinerung erschweren. Darüber hinaus wurde die monetäre Bewertung von Heil- und Hilfsmitteln unterlassen. Grund dafür war die eingeschränkte Qualität und Verwertbarkeit dieser ausschließlich vom Patienten dokumentierten Angaben sowie die fehlende Möglichkeit zur späteren Quelldatenverifizierung. Die Angaben konnten seitens des Studienmanagements daher nicht hinterfragt, ergänzt oder interpretiert werden.

Einschränkungen ergeben sich auch im Hinblick auf die Repräsentativität der erhobenen Daten und die Übertragbarkeit auf die gegenwärtige Behandlungssituation. Dies ist insbesondere durch die Teilnahme von niedergelassenen Urologen und ihrer Patienten zu erklären. $\mathrm{Zu}$ dem gab es während der laufenden Studie die Einführung einer Leitlinie, die mögliche Veränderungen in der Zuordnung zu einer Behandlungsgruppe bewirkt hat.

\section{Kurative Therapiekonzepte}

Patienten sollten bei einer Lebenserwartung $>10$ Jahre kurativ behandelt werden, ansonsten bietet sich die Palliation (WW, HT) an [5]. Kurative Therapien wie RP und RT führen zu höheren Gesamtkosten, die teils einer medizinischen Erklärung bedürfen. RP wird unter stationären Bedingungen durchgeführt; in den meisten Fällen schließt sich eine stationäre Anschlussheilbehandlung (AHB) an. Die dann folgende Tumornachsorge sieht in den ersten 2 Jahren die vierteljährliche und in den Jahren 3 sowie 4 die halbjährliche PSA-Kontrolle vor. Ab dem 5. Jahr wird PSA jährlich kontrolliert [5]. Der größte Teil der direkten Kosten entsteht also innerhalb des ersten Jahres der Therapie. RP kann offen-chirurgisch, laparoskopisch oder robotisch durchgeführt werden. Bei Einsatz des Roboters fallen Zusatzkosten im Vergleich zur offen-chirurgischen Technik in Höhe von 1375-5195 € je nach Einsatzhäufigkeit an [24]. In einer retrospektiven Analyse waren die Ergebnisse für robotisch operierte Patienten in der R0-Situation sowie bei den perioperativen Komplikationen signifikant besser [21]. Die mit dem Robotereinsatz verbundenen Mehrkosten werden bisher nicht im DRG-System abgebildet. Es ist aber damit zu rechnen, dass sie in Zukunft durch die Kostenträger übernommen werden. Damit muss es zu einer Neueinschätzung der Folgekosten nach RP aus Sicht der Kostenträger kommen. Bei einem Vergleich zwischen offener und robotischer radikaler Prostatektomie gibt es allerdings Hinweise auf eine schnellere Wiederaufnahme der Erwerbstätigkeit zugunsten der robotergestützten Vorgehensweise [23].

Obwohl die RT nach den S3-Leilinien ein onkologisch gleichwertiges Therapieverfahren ist [5], wurden in der HAROWStudie nur 383 (16\%) Patienten so behandelt. Ähnlich wie bei der RP entstehen die meisten Kosten im ersten Jahr durch die Intervention selbst. Die Nachsorge unterscheidet sich nicht. Die direkten Kosten für diese Verfahren wurden mit $1115 €$ berechnet und liegen damit deutlich unter denen der RP (7628 €). Aus den verfügbaren Daten kann die Bestrahlungstechnikjedoch nicht abgelesen werden. In den letzten Jahren zeigte sich aber ein Trend weg von der preiswerteren 3D-Conformalen (SBRT) hin zur intensitätsmodulierten Technik (IMRT) oder sogar zur Protonentherapie (PT), die deutlich kostenintensiver sind. Er- hielten im Jahr 2000 nur 28,7 \% der Patienten eine IMRT, wuchs der Anteil auf 81,7\% im Jahr 2008 [20]. Im Jahr 2010 stellte die „Agency for Healthcare Research and Quality“ (AHRQ) fest, dass es keine Evidenz dafür gäbe, dass eine bestimmte Bestrahlungsform beim PCa überlegen sei [1]. Unter dieser Annahme konnte gezeigt werden, dass die SBRT eine kosteneffektive Therapie in Bezug auf das Lebensqualitätskorrigierte Überleben im Vergleich mit der IMRT und der PT ist [22]. Im Gegensatz dazu war bei einer Dosis von >70 Gy die IMRT zwar initial teurer, aber aufgrund eines günstigeren Nebenwirkungsprofils langfristig kosteneffektiver [29]. Bei Patienten der Hochrisikogruppe empfiehlt die Leitlinie eine neo-/adjuvante Hormontherapie für 6-36 Monate. Bei der Gruppe mit mittlerem Risiko kann sich sowohl für als auch gegen eine flankierende Hormontherapie entschieden werden [5]. In der HAROW-Studie erhielten 113 Patienten zusätzlich zur RT eine HT. Die Daten lassen bei einer Nachbeobachtungszeit von 28,5 Monaten eine adjuvante Indikation mit einer Therapiedauer von 9-50 Monaten erkennen. Somit sind die direkten Kosten der adjuvanten HT denen der RT in den Folgejahren hinzuzurechnen und verursachen damit potenziell auch höhere Langzeitkosten.

Ziel von AS ist es, eine lokale Therapie möglichst lange aufzuschieben bis sie notwendig wird und bestenfalls unterlassen werden kann. Im Gegensatz zur RP und RT fallen die höchsten Kosten hier nicht im ersten Jahr der Diagnose an, sondern während der fortdauernden medizinischen Betreuung (in den ersten beiden Jahren vierteljährliche und im weiteren Verlauf halbjährliche PSA-Kontrolle und digital rektale Untersuchung). Dieser diagnostische Umfang gleicht den Nachsorgeempfehlungen nach RT und RP. Zusätzlich soll nach 6 Monaten eine Rebiopsie erfolgen. Im weiteren Verlauf soll die Biopsie zunächst im 12- bis 18-MonatsRhythmus später alle 36 Monate wiederholt werden [5]. Die Gesamtkosten von $1358 €$ setzen sich aus $375 €$ indirekten und $982 €$ direkten Kosten zusammen. Der größte Teil der direkten Kosten sind mit $702 €$ stationäre Behandlungskosten. Dieser hohe Anteil einer eigentlich am- 
Hier steht eine Anzeige.

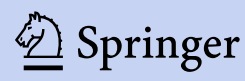


bulanten Therapie erklärt sich aus dem 20,8\%igen Anteil an pT1a- und pT1bTumoren ( $\bullet$ Tab. 1), die wegen einer Obstruktion bei Beschwerden durch ein benignes Prostatasyndrom (BPS) operiert werden. Der Anteil der unter 70-Jährigen in dieser Gruppe beträgt $55 \%$ und liegt damit zwischen RP und RT. Es muss davon ausgehen werden, dass ca. $25 \%$ der AS-Patienten innerhalb von 5 Jahren einer RP oder RT zugeführt werden [7]. In der HAROW-Studie werden die leitliniengerechten Voraussetzungen für eine AS theoretisch bei $45 \%$ der RT- und $40 \%$ der RP-Patienten erfüllt. Der häufig überbewertete Vorteil der Operation (oder Bestrahlung) wird relativiert durch den geringen tumorspezifischen Überlebensvorteil, der nur in der Altersgruppe $<65$ Jahre signifikant ist [2].

\section{Palliative Therapiekonzepte}

Watchful Waiting (WW) und Hormontherapie (HT) sind Therapieformen für Patienten mit fortgeschrittenem PCa oder solche die aufgrund ihres Alters bzw. Begleiterkrankungen voraussichtlich nicht am PCa sterben werden. Diese Therapieformen richten sich aber nicht primär an Patienten mit lokal begrenztem, also heilbaren, PCa. Sinnvollerweise wären diese Patienten erst gar nicht der PSA-gestützten Früherkennung zugeführt worden. Die richtige Indikationsstellung ist schon im Rahmen der Früherkennungssituation entscheidend. Schon bei der Aufklärung vor Bestimmung des PSA-Werts sollten sich Patient und Arzt darüber klarwerden, welche Konsequenzen aus einem suspekten Testergebnis gezogen werden und ob diese sinnvoll vor dem tatsächlichen Risiko des tumorspezifischen Todes sind. Die Gesamtkosten dieser Strategien belaufen sich in der vorliegenden Analyse auf $1929 €$ für WW bzw. $1033 €$ für HT. Insbesondere die Medikamentenkosten in der HT-Gruppe scheinen vor dem Hintergrund der aktuellen Marktpreise für Hormontherapiepräparate vergleichsweise niedrig. Das vorliegende Patientenkollektiv mit lokal begrenzten PCa hatte keine klare Indikation für eine Hormontherapie. Diese wurde entweder im Rahmen einer
Strahlentherapie in adjuvanter Indikation oder palliativ angewendet. Im Falle der adjuvanten Therapie erklärt sich die Kostendiskrepanz dadurch, dass diese Patientengruppe die Hormontherapie kürzer als 12 Monate erhalten hat. Für Patienten mit palliativem Ansatz ist die intermittierende Androgendeprivation (IAD) ein häufiges Therapiekonzept. Durch dieses Konzept sinken auch die Jahrestherapiekosten. Dennoch ist nicht komplett auszuschließen, dass die eingenommenen Medikamente nicht in der gewünschten Vollständigkeit dokumentiert worden sind. Dies gilt insbesondere für Patienten denen die entsprechenden Präparate als Depot verabreicht wurden (in Klinik oder Praxis). In diesen Fällen wäre es möglich, dass durch den Patienten keine adäquate Dokumentation dieses Arzneimittels vorgenommen wurde, da es sich um keine klassische Verschreibung handelte.

\section{Perspektive der Behandler}

Im Idealfall sollte ein Gesundheitssystem jedem Patienten die optimale Versorgung unabhängig von den entstehenden Kosten garantieren. Was jedoch eine optimale Versorgung bedeutet, ist umstritten [19]. Auf der einen Seite fordern Patienten eine umfangreiche Diagnostik, auf der anderen Seite steht das wirtschaftliche Interesse des Behandlers bzw. des Krankenhausträgers. Gerade die AS ist ein sehr betreuungsintensives Therapieverfahren. Das bedeutet, dass jeder Patient erhebliche Zeitressourcen des Arztes bindet. Dem stehen die limitierten Erlöse aus der ambulanten AS-Leistung gegenüber. In der HAROW-Studie konnten wir für die ambulante Behandlung in einem Jahr Kosten von $98 €$ ermitteln. Aus Sicht des niedergelassenen Arztes ist somit eine kostendeckende Betreuung nicht möglich. Noch drastischer fällt die Betrachtung im stationären Bereich aus. Auch hier wird leitliniengerechte Versorgung erwartet, andererseits ist das Unternehmen Krankenhaus wirtschaftlich zu führen. Das kann möglicherweise dazu führen, dass Therapieentscheidungen auch durch betriebswirtschaftliche Interessen beeinflusst werden. Grundsätzlich zeigen die Ergebnisse der HAROW-Studie tatsächlich teils deutliche Abweichungen von heutigen Leitlinienvorgaben. Dies ist allerdings auch zu erklären mit dem Zeitpunkt der Einführung der „Interdisziplinären Leitlinie der Qualität S3 zur Früherkennung, Diagnose und Therapie der verschiedenen Stadien des Prostatakarzinoms" während der laufenden HAROWStudie. Da zu Beginn der Studie 2008 noch keine Leitlinie existierte und da es sich um eine Studie aus dem Bereich der Versorgungsforschung handelt, entsprechen die Therapien nur bedingt den heutigen Vorgaben. Prinzipiell liegt der Wert von Versorgungsforschungsansätzen wie in der HAROW-Studie auch und gerade in der Offenlegung solcher Differenzen.

\section{Ausblick}

Die Analyse macht deutlich, dass die Behandlung des PCa erhebliche ökonomische Folgekosten nach sich ziehen kann. RP war sowohl aus gesellschaftlicher Sicht als auch aus Sicht der Krankenkassen in Deutschland mit den höchsten ökonomischen Lasten assoziiert. Die direkten Kosten scheinen für AS, RT, HT und WW vergleichbar zu sein. Hinsichtlich der indirekten Kosten sind die Ergebnisse unsicher, da altersbedingt nur noch wenige Patienten erwerbstätig sind. Deutlich wird die Notwendigkeit zur Durchführung von gesundheitsökonomischen Langzeitstudien, die behandlungsspezifischen Unterschieden im zeitlichen Auftreten von Kosten besser gerecht werden.

\section{Fazit für die Praxis}

- Die gesundheitsökonomischen Ana-
lysen der HAROW-Studie bieten
einen transparenten Blick auf die
Ressourceninanspruchnahme und
Kosten unterschiedlicher Strategien
zur Behandlung des PCa im deut-
schen Versorgungsalltag. Zudem gibt
die Analyse Hinweise auf mögliche
Einsparpotenziale sowie auf ökono-
mische Fehlanreize in der derzeitigen
Versorgung von PCa-Patienten im
deutschen Gesundheitssystem.
- Therapeuten sollten sich bei der
Auswahl eines Behandlungsregimes
der stark differierenden Folgekosten
für Gesellschaft und Kostenträger


bewusst sein, ohne ihre Therapieentscheidung allerdings allein davon abhängig zu machen.

- Vor dem Hintergrund indirekter Folgekosten, insbesondere durch Arbeitsunfähigkeit und frühzeitige Berentung, sollten die behandelnden Ärzte bei ihrer Therapieentscheidung auch das Alter und den Erwerbstatus des Patienten berücksichtigen.

\section{Korrespondenzadresse}

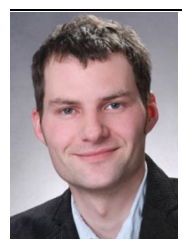

PD Dr. rer. medic.

T. Reinhold

Institut für Sozialmedizin,

Epidemiologie und

Gesundheitsökonomie,

Charite - Universitätsmedizin Luisenstr. 57, 10117 Berlin, Deutschland thomas.reinhold@charite.de

Danksagung. Wir danken allen Patienten und Ärzten für ihre Teilnahme an der dieser Analyse zugrunde liegenden HAROW-Studie. Die HAROWStudie wurde finanziell unterstützt durch GAZPROM Germania $\mathrm{GmbH}$.

\section{Einhaltung ethischer Richtlinien}

Interessenkonflikt. T. Reinhold und C. Dornquast geben an, dass kein Interessenkonflikt besteht. C. Börgermann ist Mitglied in der Deutschen Gesellschaft für Urologie und der Expertengruppe S3-Leitlinie Prostatakarzinom. L. Weißbach gibt seine Beratertätigkeit bei der Stiftung Männergesundheit an und ist zudem Mitglied des WIdO-Panels "Qualitätssicherung" der AOK.

Alle beschriebenen Untersuchungen am Menschen wurden mit Zustimmung der zuständigen Ethik-Kommission, im Einklang mit nationalem Recht sowie gemäß der Deklaration von Helsinki von 1975 (in der aktuellen, überarbeiteten Fassung) durchgeführt. Von allen beteiligten Patienten liegt eine Einverständniserklärung vor.

Open Access. Dieser Artikel wird unter der Creative Commons Namensnennung 4.0 International Lizenz (http://creativecommons.org/licenses/by/4.0/deed. de) veröffentlicht, welche die Nutzung, Vervielfältigung, Bearbeitung, Verbreitung und Wiedergabe in jeglichem Medium und Format erlaubt, sofern Sie den/die ursprünglichen Autor(en) und die Quelle ordnungsgemäß nennen, einen Link zur Creative Commons Lizenz beifügen und angeben, ob Änderungen vorgenommen wurden.

\section{Literatur}

1. Bannuru RR, Dvorak T, Obadan N et al (2011) Comparative evaluation of radiation treatments for clinically localized prostate cancer: an updated systematic review (DARE structured abstract). Ann Intern Med 155:171-178

2. Bill-Axelson A, Holmberg L, Garmo H et al (2014) Radical prostatectomy or watchful waiting in early prostate cancer. NEngl J Med 370:932-942

3. Bock J, Brettschneider C, Seidl H et al (2015) Ermittlung standardisierter Bewertungssätze aus gesellschaftlicher Perspektive für die gesundheitsökonomische Evaluation. Gesundheitswesen 77:53-61

4. Bundesvereinigung Deutscher Apothekerverbände (2010) ABDA-Artikelstamm (Taxe). Bundesvereinigung Deutscher Apothekerverbände, Eschborn

5. Deutsche Krebsgesellschaft (2014) Leitlinienreport Interdisziplinäre Leitlinie der Qualität S3 zur Früherkennung, Diagnose und Therapie der verschiedenen Stadien. Langversion 3.1-2. Aktualisierung-Oktober 2014

6. Dragomir A, Cury FL, Aprikian AG (2014) Active surveillance for low-risk prostate cancer compared with immediate treatment: a Canadian cost comparison. CMAJ Open 2:E60-E68

7. Eggener SE, Mueller A, Berglund RK et al (2014) A multi-institutional evaluation of active surveillance for low-risk prostate cancer. J Urol 181:1635-1641

8. Eldefrawy A, Katkoori D, Abramowitz $M$ et al (2013) Active surveillance vs. treatment for lowrisk prostate cancer: A cost comparison. Urol Oncol Semin Orig Investig 31:576-580

9. German Centre for Cancer Registry Data (2016) Database query incidence prostate cancer. http:// www.krebsdaten.de. Zugegriffen: 13. Januar 2016

10. Gesundheitsberichterstattung des Bundes (2015) Krebs in Deutschland 2011/2012 10 Bd. 10. Robert Koch Institut, Berlin

11. Herden J, Ansmann L, Ernstmann N et al (2016) The treatment of localized prostate cancer in everyday practice in Germany - A multicenter prospective observational study (HAROW) in 2957 patients. Dtsch Arztebl Int 113:329-336

12. Holmberg L, Bill-Axelson A, Steineck G et al (2012) Results from the scandinavian prostate cancer group trial number 4: A randomized controlled trial of radical prostatectomy versus watchful waiting. J Natl Cancer Inst Monographs 2011:230-233

13. Keegan KA, Dall'Era M, Durbin-Johnson B, Evans CP (2012) Active surveillance for prostate cancer compared with immediate treatment - an economicanalysis. Cancer 118(14):3512

14. Krauth C, Hessel F, Hansmeier T et al (2005) Empirical standard costs for health economic evaluation in Germany - a proposal by the working group methods in health economic evaluation. Gesundheitswesen 67:736-746

15. Laviana AA, Ilg AM, Veruttipong D et al (2016) Utilizing time-driven activity-based costing to understand the short- and long-term costs of treating localized, low-risk prostate cancer. Cancer 122(3):447-

16. Liljas B (1998) How to calculate indirect costs in economic evaluations. Pharmacoeconomics 13:1-7

17. Mariotto AB, Yabroff KR, Shao $Y$ et al (2011) Projections of the cost of cancer care in the United States: 2010-2020. J Natl Cancer Inst 103:117-128
18. Matsumoto K, Haga K, Kitazawa T et al (2015) Cost of illness of breast cancer in Japan: trends and future projections. BMC Res Notes 8:539

19. Molinier L, CastelliC, BauvinEetal (2011) Coststudy of the clinical management of prostate cancer in France: Results on the basis of population-based data. Eur JHeal Econ 12:363-371

20. Nguyen PL, Gu X, Lipsitz SR et al (2011) Cost implications of the rapid adoption of newer technologies for treating prostate cancer. J Clin Oncol 29:1517-1524

21. Niklas C, Saar M, Berg B et al (2015) da Vinci and open radical prostatectomy: comparison of clinical outcomes and analysis of insurance costs. Urol Int 96(3):287-294

22. Parthan A, Pruttivarasin N, Davies D et al (2012) Comparative cost-effectiveness of stereotactic body radiation therapy versus intensity-modulated and proton radiation therapy for localized prostate cancer. FrontOncol 2:81

23. Plym A, Chiesa F, Voss M et al (2016) Work disability after robot-assisted or open radical prostatectomy: A nationwide, population-based study. Eur Urol 70:64-71

24. ProbstM,BentasW, Wolfram Metal(2006)Radikale Prostatektomie mit dem DaVinci System unter DRG -Bedingungen. Aktuelle Urol 37(2):102

25. Reinhold T, Brüggenjürgen $B$, Schlander $M$ et al (2010) Economic analysis based on multinational studies: methods for adapting findings to national contexts. J Public Health 18:327-335

26. Roehrborn CG, Black LK (2011) The economic burden of prostate cancer. BJU Int 108:806-813

27. Stokes ME, Ishak J, Proskorovsky I et al (2011) Lifetime economic burden of prostate cancer. BMC Health Serv Res 11:349

28. Wilt TJ, Brawer MK, Jones KM et al (2012) Radical prostatectomy versus observation for localized prostate cancer. N Engl J Med 367:203-213

29. Yong JHE, Beca J, McGowan T et al (2012) Costeffectiveness of intensity-modulated radiotherapy in prostate cancer. Clin Oncol 24:521-531 